\title{
Re-Engineering Collaborative Practice in Primary Care: Integrating Community Pharmacy to the Clinic; Creating a Pharmacy Referral and Quality Circle
}

\author{
Jimmy Shangala Mwawaka, RPh
}

Kenya Medical Research Institute, Student of Biotechnology, Innovation and Regulatory Science at Purdue University

\begin{abstract}
Pharmacy practice is part of a value chain made up of suppliers(S), inputs(I), processes(P), outputs(O), and customers(C). The interface between community pharmacies and clinic-based prescribers is complicated by challenges related to gaps in the design of the chain. The supplier-input-process-outcome-customer (SIPOC) model can be used to re-engineer the chain: integrating an intervening clinical pharmacist in the interface creates a structure for interprofessional collaboration and communication across the interface. This innovation has important implications for patient referral between clinic and pharmacy, and the future role and scope of all patientfacing pharmacists.
\end{abstract}

Keywords: pharmacy referral; community pharmacy; collaborative care; process re-engineering; primary care; quality circle; integration

\section{BACKGROUND}

Community pharmacists enjoy considerable autonomy and have the greatest potential to deliver pharmaceutical care. To provide care, the pharmacist needs the consent of the patient or the patient's caregiver. If pharmaceutical interventions are required, the pharmacist must seek the concurrence or the involvement of the prescriber in implementing care plans.

Process re-engineering is a technique of quality improvement which involves simplifying, adding, subtracting, expanding, shortening, replacing, combining, or rearranging any number of steps in a process chain for delivery of a given product or service. The goal is to create synergy and to increase productivity, efficiency, effectiveness, and quality.

Drug-related patient care delivery and experience is a valuechain consisting of suppliers(s), inputs(i), processes(p), outputs(o) and customers(c)-SIPOC. Delivery of medicines to patients, which is a core process of pharmacy practice, can be analyzed using the Sipoc model. Activities that are critical to or that facilitate the satisfaction of customer requirements are termed value-added or value-adding. Those that do not enhance customer satisfaction are categorized as non-valueadding. The latter is the usual target of process re-engineering.

Regarding pharmaceutical products, the minimum requirements of the pharmacy customer are product safety, identity, potency, purity, and quality. Pharmacists and other supply chain professionals add value to the finished pharmaceutical product by legally procuring it; safely

Corresponding author: Jimmy S. Mwawaka, RPh

Hospital and Clinical Research Pharmacist

Kenya Medical Research Institute

Student of Biotechnology, Innovation and Regulatory Science

at Purdue University

P.O Box 23980108 Kilifi, KENYA

Tel $+254-714929286$ distributing it; providing appropriate product packaging and dose-delivery devices; by safeguarding the product against misuse, diversion, wastage adulteration, and deterioration; by ascertaining and assuring its quality and integrity to the point of use; by imparting relevant information, knowledge, and skills to assure its effective use; by optimizing access to the product; by preventing the influx of substandard and/or falsified medicines into the supply chain; and by studying and monitoring its use and outcomes.

\section{Collaborative Models of Clinical Pharmacy Practice}

Within health facilities, clinical pharmacists work alongside physicians and other clinical staff. Pharmacist access to the patient's medical record is essential to pharmacist-physician collaboration in primary care (Bloodworth, Malinowski, Lirette, and Ross; 2019). Several models of such interprofessional collaboration have been institutionalized:

1. Globally, the predominant model of practice has clinical pharmacists as members of the multidisciplinary, physician-led, hospital ward-based teamthe pharmacist acts as the pharmaceutical expert to the lead physician who makes the final decision on what medicine or dose, gets prescribed, started, terminated, or modified.

2. In the United Kingdom, pharmacists collaborate formally with physicians, nurses, and clinic staff in the following roles:

- Practice-based pharmacist: This pharmacist manages the medicine use process and participates in direct care within a given medical practice.

- Prescribing advisor: This pharmacist participates in long-term planning activities to support medicine use and management in a consortium of group medical practices. 
- Non-medical prescriber: This pharmacist may work as a supplementary or independent prescriber. As independent prescribers, pharmacists can prescribe any licensed medicine (other than controlled medicines) for any ailment within the limits of their own competence. Both supplementary and independent pharmacist prescribers work in medical practices and community pharmacies. Efforts have been made to establish pharmacist-led clinics and to create positions in all areas of hospital care including critical care (Hill, Marr, and Smith,2019).

3. In the United States of America (USA), pharmacists sign collaborative practice agreements (CPAs) with physicians for collaborative practice within a given therapeutic area. The contract creates a referral system between the pharmacist and the physician or other service providers. The pharmacist need not be based within the physician's practice.He/she can carry on other work or sign other CPAs with other physicians.

4. In Australia, the aged care facility pharmacist and the general practice pharmacist are established advanced practice roles. The pharmaceutical society of Australia (PSA) recognizes "liaison with community pharmacy to enable hospital-to-community transition of care "as one of the patient-level activities within the role of a geriatric facility pharmacist.

5. In the USA, medication therapy management (MTM) is a statute-mandated pharmacist-provided healthcare service. MTM is provided by pharmacists within an ambulatory clinic or community pharmacy.

\section{Community Pharmacy}

Hawksworth, Corlett, Wright, and Chrystyn (1999) concluded that interventions undertaken by community pharmacists during the dispensing process have the potential to improve health outcomes.But, the community pharmacist and the community-based prescriber are independent of each other. Interestingly, patients prefer that pharmacists and physicians work closely together (Mc Donough, 2012) in planning and implementing pharmaceutical care plans.

\section{Barriers to Implementation of Pharmaceutical Care in Community Pharmacy}

Collaboration between medical clinics and community pharmacies would benefit the clinic, the pharmacy, the patients, and the payers (Doucette, 2019).

The following challenges impede such collaboration:

i. Conflict of interest: There is a conflict of interest between the professional and business aspects of community pharmacy (Goode, Owen, Page and Gatewood, 2019). ii. Relationship building: Community pharmacists (even when clinically trained) lack a continuous professionalclient relationship with the patient. Whereas patients work with a few physicians and health centers on a regular basis, they frequently patronize several different pharmacies and interact with different staff at those facilities.

iii. Perception: Often clinical interventions by the pharmacist are perceived as an intrusion on the patient-physician relationship, and even a non-valueadding complication to care. Suggestions made by the pharmacist may not be communicated effectively or in good time, and uptake of pharmacist advice is uncertain (Loeffler et al,2017). Loeffler et al (2017) identified lack of trust as a leading barrier to interprofessional collaboration between physicians and pharmacists. Additional barriers were lack of communication structures and discordance about the clinical importance of pharmaceutical information.

iv. Information flow: Pharmacist interventions are rarely recorded, and even when recorded are rarely shared with the national health authorities. Communication between dispensing pharmacists and prescribers is delayed, slow and difficult-and frequently queries are relayed indirectly to the physician through assistants and secretaries.

v. Infrastructure: Pharmacies and clinics lack a framework for collaboration in the provision of patient care. This may be due to the traditional boundaries that exist between pharmacy and medicine. Other factors include lack of communication channels and competing interests.

Gaps in the design of clinic-community pharmacy interactions

- Independently, both the clinical and community pharmacist, as currently established, are ineffective in meeting the needs of the patient. A clinical pharmacist who practices away from the traditional pharmacy lacks the wisdom and know-how of the pharmaceutical world and cannot, therefore, deliver the total value of pharmaceutical insight to patient care. Conversely, a traditional community pharmacist is deficient in clinical insight and cannot, therefore, provide effective clinical services.

- Detecting, correcting, and resolving preventable problems and errors are non-value adding activities-a pharmacist's input into drug prescribing is more useful if available at the time of prescribing or earlier.

- Designing and planning interventions that cannot be implemented is a non-value-adding activity stream. Pharmacists cannot effectively contribute to patient care if commitment on the part of physicians, patients, and other collaborating parties to implement pharmaceutical interventions is not guaranteed and entrenched in the patient care value chain. 
- Effective communication between pharmacists and physicians frequently seems to be a disruptive, lowpriority, and reactive activity. As to when, how, if, and where this activity occurs is often left to chance. Information flow- in both directions- between the two parties should be planned, structured, and optimized through process design and planning.

- Interprofessional collaboration fails due to a lack of a supportive structure for decision-making, accountability, and governance. Howard et al (2003) identified "the need to refine the referral process and to work out professional role relationships and ongoing collaboration more fully". Attempts at interprofessional collaboration fail to create or consolidate professional interdependence. The world health organization (WHO) holds that "the first challenge in professional integration is to have the appropriate range of skills available in the health service; the second challenge is to ensure that different professional groups collaborate effectively."

\section{New thinking}

McDonough (2016) suggests that a shift in community pharmacy from a dispensing model to a service model is essential to meeting the demand for clinical pharmacy services. For effect, the interaction between the prescribing physician and the consulting clinical pharmacist should be structured i.e. designed. Bollen, Harrison, Aslani and van Haastregt (2019) identified the physical environment, co-location, and resources -to facilitate clear and regular communication- as important facilitators of collaboration between general practitioners and community pharmacists:

\section{- Pharmacists as quality champions:}

A study found that non-dispensing pharmacists (NDPs) ,integrated into general medical practices, are more successful where they have a distinct role and where their work is 'framed as" quality management (Hazen et al, 2019). By reviewing the work done by the physician, clinical pharmacists act as process auditors. In this role, their work involves identifying physician outputs that require reprocessing or reworking. It is possible to empower the practice-based pharmacist to correct identified defects.

- Recognizing the voice of the consumer:

The current patient-care process requires cooperation of two important subsystems i.e. prescribing and dispensing. These subsystems encompass two separate sets of activities and responsibilities. Thus, the patient is expected to consult both the physician and the pharmacist, and deal with the anxiety of one professional reviewing or correcting decisions made by the other. Worse still access to the benefit of pharmacist interventions depends on the goodwill of the physician and the consent of the patient. Occasionally, the patient is pushed to choose to align with one party to the exclusion of the other. This burden on the customer makes pharmaceutical care services both untenable and ineffective. Patients would experience better care if these two sub-systems were fashioned into a single seamless system. New approaches are required to get the physician and pharmacist to collaborate without any burden to the patient.

- Pharmacists working in teams and through teams: Community pharmacists serve their customers through technicians and pharmacy assistants. Thus, auxiliary pharmacy staff act as pharmacist extenders. By the same analogy, practice-based clinical pharmacists can serve as physician extenders. In the same vein, pharmacists working with medical insurance and HMO firms frequently manage the performance of community pharmacists to ensure compliance with prescribing controls. One can envision community pharmacists becoming extenders of institution-based clinical pharmacists.

- Referral channels: Zuckerman et al (2019) described the emerging role of the integrated specialty pharmacist who coordinates care during transitions across care sites and across provider types within a given site. Keely et al (2016) found that involving pharmacists in referral decisions improves outcomes of therapy during or after transitions. To move community pharmacy forward, we need to put in place a formal referral channel between the clinic and the community pharmacy.

- Rethinking professional autonomy: Professionals need only as much autonomy as is necessary to serve the best interests of the client. To gain more say in matters of patient care, community pharmacists will have to forgo some of their current professional autonomy. This is the essence of collaborative care. This pays dividends in terms of greater pharmacist integration into the health system and better patient outcomes.

- Restructuring of collaborative practice: In their study of nurse practitioners, Côté et al (2019) held that effective collaboration involves the redefinition of roles, and the creation of new work environments and structures.Physician-pharmacist collaboration will lead to a redefinition of traditional boundaries and roles for both physicians and pharmacists.

- Stakeholder buy-in: Adamcik, Ransford, Oppenheimer, Brown, Eagan, and Weissman (1982) found that physicians and nurses were more favorable to pharmacists making clinical decisions in the hospital than in the community setting. We should, therefore, seize every opportunity to strengthen the role of the clinic-based clinical pharmacist. Conversely, community pharmacy expansionists should put in place measures to assure the quality of clinical interventions undertaken by pharmacists in community pharmacy. 
- Healthcare without borders: Mossialos et al (2015) underscore the need to integrate community pharmacists in primary care and to develop "tiered" pharmacist services. Integrating clinics with community pharmacies assures patient, service, and information flow in both directions. This promotes the efficiency and effectiveness of services.

- Interprofessional accountability: Institutions that contract out work have a responsibility to control the performance of the contract acceptor. Providers (and by extension staff in health facilities) who issue prescriptions to patients have a right to insist that those prescriptions be dispensed faithfully and without errors by community pharmacists. Pharmacists who rely on clinics and hospitals (S) for prescriptions have a legitimate expectation that those prescriptions would be free of errors. It is therefore acceptable that clinic staff should manage the performance of "contracted" pharmacies. A prescriber has a role in determining what happens to "his/her prescription ". Clinics would do well to invest in capabilities that could assure the delivery of flawless prescriptions.

- Addressing the no-man's territory: Between the clinicbased prescriber and the dispensary-based pharmacist is a professional desert-a big knowledge, skill, culture, and practice gap- that slows down interprofessional work. This no-man's territory needs to be filled by a professional versed in the two sub-systems. Using a clinical or clinic-embedded pharmacist at this interface, with a view to integrating the pharmacy into the clinic, can thin out the barrier.

- Quality by design: In traditional production systems, producers achieve quality by identifying and weeding out defective or non-conforming products. This they do largely by inspecting and testing a sample of products from each production batch. This is both wasteful (non-conforming products are destroyed, reworked, or sold off cheaply) and labor-intensive. The modern approach to quality uses knowledge of process risks to design errors and defects out of the production process. Pharmaceutical interventions, as currently done, represent a practice based on the inspection approach. To be proactive in preventingrather than detecting-medical errors, clinical pharmacists ought to engage with prescribers before the prescription is written out and/or released to the dispensing pharmacist.

\section{PROPOSAL}

\section{Concept}

Coupling community pharmacists with institutional clinical pharmacists could enhance and synergize the value of both pharmacists to the patient care value chain. The clinical pharmacist would practice at the interface of the clinic and the pharmacy, providing a link between the two health settings. This would re-structure the prescriber-dispenser relationship in a fundamental way.

In the proposed model:

1. the clinical pharmacist would act as a moderator between the community pharmacy and the clinic. Both the community pharmacy and the clinic are customers (C) to the clinical pharmacist for whom the clinical pharmacist generates outputs (O).

2. the clinical pharmacist would mediate and advocate for the clinic (physician and his team or groups of prescribers); the patient and his/her family or caregivers; and the dispensing community pharmacist and his/her firm. These 3 customers (C) to the clinical pharmacist would also supply informational and demand Inputs (I) to the clinical pharmacist.

3. community pharmacist would channel drug-related information, reports of Drug Use Reviews, reports of adverse drug reactions and events, concerns about non-adherence, and other drug-related problems, prescription queries, and suggestions for change of therapy to the clinical pharmacist for review and further processing. Thus both parties would be engaged in alternate -if not simultaneous-exchanges as customers (C) and suppliers (S).

4. the clinical pharmacist would capitalize on his longterm alignment with his suppliers, the prescriber(s), to secure prompt consideration and effective implementation of all pharmaceutical interventions.

\section{Workflow and distribution}

Sequence: In the proposed model the prescriber consults with a patient in the presence of or in collaboration with a resident or remote clinical pharmacist. The clinical pharmacist would review and endorse the prescription in real-time before it is printed out to the patient or relayed to the dispensing pharmacist.

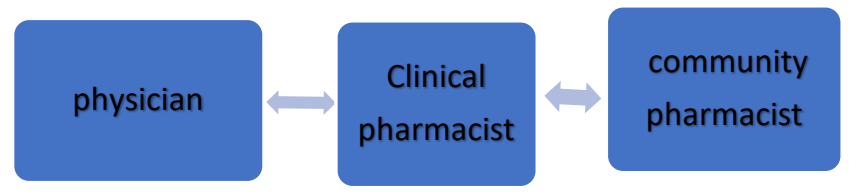

Fig 1: patient and information flow through the clinical pharmacist from both the prescriber and the community pharmacist. 
Responsibility: The clinical pharmacist will make decisions on drug-related problems and update the patient medical record on behalf of the physician. He will also offer support supervision to the community pharmacist, and the latter will supply the former with commercial, product-related information and expertise.

Referrals: the clinical pharmacist may recommend to patients a pharmacist-led service that can be delivered by the community pharmacist. The community pharmacist may initiate care for a patient and undertake medicine use review; any cases that require more extensive pharmacist evaluation and intervention may be referred to the clinical pharmacist.

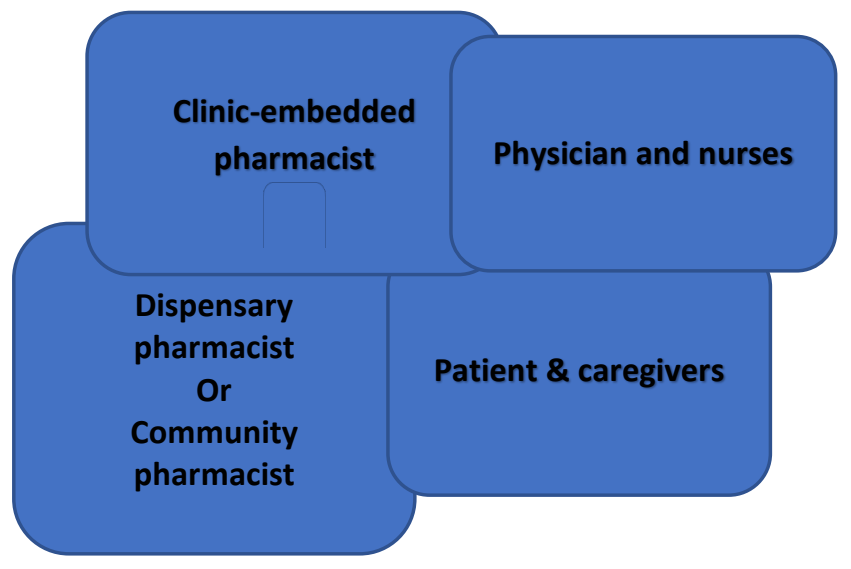

Fig 2: A clinic-embedded pharmacist moderating interactions amongst physician, nurse, clinic dispensary pharmacist, community pharmacist, patient, and caregivers.

\section{DISCUSSION}

\section{Innovation}

This proposed innovation seeks to extend the American CPAfacilitated model and the UK's national health service (NHS) pharmacist model of collaborative practice. The model uses the clinical pharmacist to improve clinical outcomes and customer satisfaction at the community pharmacy level. In the proposed innovation, the clinic-based (clinical) pharmacist navigates the patient's interaction with the community pharmacist. With the benefit of access to the prescriber and/or the patient's file, the clinical pharmacist will perform a clinical check of a prescription and endorse it before it reaches the (community) pharmacy. The model proposes a structured collaboration between the clinical pharmacist (clinic) and the community pharmacist; there is communication and patient flow in both directions.

Commenting on the Australian landscape, Freeman, Rigby, and Williams (2019) suggested that the distinctive knowledge and skills of pharmacists can be deployed to prevent fragmentation of care and to reduce medication misadventure in primary care. The proposed model endorses this position with significant improvements: i. collaboration is between two independent pharmacists.

ii. the community pharmacist acts as a clinical pharmacist extender: all interventions at the pharmacy level are discussed with and/or processed by the clinical pharmacist who has an ongoing relationship with both the dispensing pharmacist and the prescriber.

iii. all pharmacist interventions are entered into the patient's file monitored and evaluated by the clinical (or clinicembedded) pharmacist.

iv. the community pharmacy team and the clinic team would function as a virtual service delivery unit that would also be empowered to assure and improve quality.

v. The clinical pharmacist being versed in the two subsystems enhances both event-related and continuous communication and service planning between the clinic and the pharmacy.

vi. the need for pharmaceutical interventions at the point of sale is minimized by having the clinical pharmacist review the prescription before it reaches the pharmacy. 


\section{Justification}

- Prescribing and dispensing are linked together by their mutual goal: the delivery of medicine to the patient. Redistributing responsibility between the community pharmacist and his clinic-based colleague in the interest of positive patient outcomes is therefore justifiable. By forging a longtime alliance with the community pharmacist, the clinical pharmacist would provide a robust clinic-integrated pharmacy subsystem.

- The current role of the community pharmacist is to clarify the needs of the entity generating the prescription. The clinical pharmacist ensures that the community pharmacist receives clear, legitimate, valid, complete, safe, and rational prescriptions and ensures that the dispensing pharmacist is supported to deliver the care intended.

- This proposal seeks an incremental improvement of an established system. It creates value for the customer, assures quality at source, and seeks perfection.

\section{Benefits}

- The re-engineered process saves time spent, by the community pharmacist, in resolving prescribing and dispensing errors, designing and implementing pharmaceutical interventions and following up patients.

- The innovation addresses the barriers to pharmaceutical care in community pharmacy: conflict of interest, negative perception, relationship-building, information flow, and infrastructure. Pharmaceutical interventions will be implemented within or with the consent of the clinic. Patients will thus perceive and experience the community pharmacy as an extension of the clinic. The clinic-embedded pharmacist refers the patient to a specific community pharmacist or community pharmacy group. This fosters long-term relationship building between the community pharmacist and his/her patient.

- With the clinical pharmacist in the middle, the new process provides for a smooth transition of patients from the clinic to the community pharmacy and vice versa.

- This process can be used to refer patients from the community to the physician's clinic and vice versa.

- The rate of prescription rejection and amendment at the retail pharmacy level will decline. This will yield time savings at the pharmacy and thereby result in a more favorable customer experience.

- Pharmacy client registration and tracking will be facilitated.

- Both clinical pharmacy and community pharmacists will be strengthened: operating at the top of their respective licenses, both clinical and community pharmacists will have a regular and adequate flow of work and income. There will be a value addition to care as the prescription and/or patient moves from one pharmacist to the next.
- A communication and referral system will be created between the community-based clinic and the community pharmacy. Clinical pharmacists will have access to care information from both ends of the care continuum.

- Community pharmacists will have a greater understanding of the needs of their patients, and they will be more engaged with their patients' care. This will enhance self-esteem, self-confidence, inculcate a sense of professional purpose and lead to greater career fulfillment.

\section{Feasibility}

a. Financing: Clinical pharmacists will be reimbursed by contracting physicians. The community pharmacists will levy charges for the products and the dispensing service.

b. Acceptability: It is feasible to transfer some clinical responsibility from the community pharmacist to the clinic-based pharmacist. The proposal conserves the autonomy of the profession by strengthening the role of clinic-based (clinical) pharmacists. This pharmacist prequalifies pharmacies to which prescriptions may be referred. This is akin to the practice (ethical or otherwise) in many developing countries where pharmacy establishments go out of their way to get endorsed or recommended by prescribers. In the proposed model, the endorsement or recommendation will come from a fellow pharmacist whose decision to refer will be based on objective preestablished criteria.

c. Implementation: The proposal may be implemented in the following 5 steps:

Step 1: clinic hires or designates coordinating clinical pharmacist (CP)

Step 2: $\mathrm{CP}$ and community pharmacist agree to collaborate

Step 3: clinic and pharmacy leads formalize collaboration between clinic and pharmacy to specify financing, responsibilities, roles, and obligations; a coordinating team (Team) is appointed.

Step 4: Team develops Standard Operating Procedures for referrals, consultations, capability building, business development, governance, quality management, and problem-solving.

Step 5: scale-up to include more clinical pharmacists and community pharmacists-both clinic and pharmacy may sign agreements with other pharmacies and clinics to expand the collaboration circle. 


\section{Implications for the practice of pharmacy}

\section{Pharmacy skill development and deployment}

- Community pharmacy will require little clinical skill. Community pharmacists could therefore delegate the dispensing function to technicians and focus on quality assurance and staff development. A shorter and less expensive program would be enough for preparing community pharmacists.

- Clinic-embedded clinical pharmacists could sign up agreements for collaboration with community pharmacy-based pharmacists to scale-up certain limited pharmaceutical interventions e.g. therapeutic substitution, dose adjustment, formulation changes within a given route of administration, etc.

- The distribution (and hence the number) of both community pharmacies and pharmacists would be closely matched with that of clinics and physicians.

- Clinical pharmacists would increasingly find it difficult to practice in community pharmacy: clinical pharmacists would need to distance themselves from dispensing. This could be achieved by moving out of the pharmacy, delegating dispensing work to another pharmacist or even technician, transforming the pharmacy into an office, and charging for every consultation and advisory service.

- With time the community pharmacist would join or become the clinical pharmacist and leave more dispensing work to the technician. This would translate into greater productivity for the value chain (Kalman, Witkowski, and Ogawa, 1992).

\section{Patient referral}

- A referral-supervision system for community pharmacy would emerge. This system would feed the contribution of community pharmacists into the broader health system. Clinical data generated at the pharmacy level would be captured by the national epidemiological system as part of clinic data.

Professional identities, roles, and responsibilities

Designing the collaboration between the physician, clinical pharmacist, and community pharmacist fosters communication, consensus-building and mutual understanding on division of labor and responsibilities amongst the parties.

- Clinical pharmacists will rightly be perceived as part of the physician's team and as professionals who are primarily motivated by a desire for optimal outcomes of patient care.

- Community pharmacists will retain their identity as expert custodians of medicines but with the added role for assuring the quality of the product and its effective use. Community pharmacists will have ongoing access to the expertise of a clinical pharmacist. Practice-based pharmacists will have another role: selection, development, and supervision of contracted community pharmacies.
- $\quad$ The redefined community pharmacist would be able to supervise more than one pharmacy. This may be the solution for developing countries where there is a scarcity of pharmacists: technicians may be retrained to assume the role of the current pharmacist who, in turn, could be retrained to become the clinic-based pharmacist. Alternatively, the current community pharmacist may become superintendent community pharmacist with expanded responsibility for ensuring or reviewing supply chain and product distribution quality for several pharmacies.

- This would be an opportunity for clinical pharmacists to pilot remote working.

- $\quad$ This model creates a leadership structure in pharmacy. It provides a framework for relations between wardbased and dispensary-based hospital pharmacists within a given institution- the pharmacist in the clinical care team would lead communication and interactions between the care team and the pharmacy-based pharmacists.

\section{Pharmacy workforce in low-resource settings}

- In sub-Saharan Africa, where public hospitals frequently experience medicine stockouts, patients are often required to purchase prescribed medicines from private pharmacies. Where such prescriptions have technical or clinical issues, pharmacy staff find it difficult to trace or contact the prescriber for clarification or intervention. This leads to rejection of the contentious prescriptions, erroneous dispensing, or implementation of pharmaceutical interventions without the knowledge and consent of the prescriber. To prevent this situation, it would help if hospital pharmacists could review all outgoing prescriptions for appropriateness and assume responsibility for receiving and resolving all prescription queries from private pharmacies. In this case, all pharmacy queries would be channeled to the hospital-based pharmacist rather than the specific prescriber.

- Hospital and pharmacy department managers could go further and accredit private pharmacies as a prequalification for filling prescriptions generated by the hospitals. This would create a secure supply chain for the benefit of the patient.

\section{CONCLUSION}

Both clinical and dispensing pharmacists have legitimate roles in healthcare. Community pharmacy can be strengthened through its integration into the community clinic. This can be realized by introducing a clinic-based pharmacist at the interface of the community physician and community pharmacist. This supports continuity of care and optimizes the roles of both institutional and community pharmacists. The proposed model improves bi-directional information flow and patient referral between clinic and community pharmacy. It also has important implications for the future role and scope of all patient-facing pharmacists. 
Conflict of interest: Jimmy Shangala Mwawaka is a hospital and clinical research pharmacist in Kenya. He is also a student of Biotechnology, Innovation and Regulatory Science at Purdue University. He has neither financial interest nor conflict of interest to declare.

\section{REFERENCES}

1. Adamcik, B. A., Ransford, H. E., Oppenheimer, P. R., Brown, J. F., Eagan, P. A., \& Weissman, F. G. (1986). New clinical roles for pharmacists: a study of role expansion. Social science \& medicine (1982), 23(11), 1187-1200. https://doi.org/10.1016/0277-9536(86)90338-2

2. Bloodworth, L. S., Malinowski, S. S., Lirette, S. T., \& Ross, L. A. (2019). Pharmacist linkage in care transitions: From academic medical center to community. Journal of the American Pharmacists Association: JAPhA, 59(6), 896-904. https://doi.org/10.1016/i.japh.2019.08.011

3. Bollen, A., Harrison, R., Aslani, P., \& van Haastregt, J. (2019). Factors influencing interprofessional collaboration between community pharmacists and general practitionersA systematic review. Health \& social care in the community, 27(4), e189-e212. https://doi.org/10.1111/hsc.12705

4. Clark, C. M., LaValley, S. A., Singh, R., Mustafa, E., Monte, S. V., \& Wahler, R. G., Jr (2020). A pharmacist-led pilot program to facilitate deprescribing in a primary care clinic. Journal of the American Pharmacists Association: JAPhA, 60(1), 105-111. https://doi.org/10.1016/j.japh.2019.09.011

5. Côté, N., Freeman, A., Jean, E., \& Denis, J. L. (2019). New understanding of primary health care nurse practitioner role optimisation: the dynamic relationship between the context and work meaning. BMC health services research, 19(1), 882. https://doi.org/10.1186/s12913-019-4731-8

6. Doucette W. R. (2019). Innovative Collaboration between a Medical Clinic and a Community Pharmacy: A Case Report. Pharmacy (Basel, Switzerland), 7(2), 62. https://doi.org/10.3390/pharmacy7020062

7. Goode, J. V., Owen, J., Page, A., \& Gatewood, S. (2019). Community-Based Pharmacy Practice Innovation and the Role of the Community-Based Pharmacist Practitioner in the United States. Pharmacy (Basel, Switzerland), 7(3), 106. https://doi.org/10.3390/pharmacy7030106

8. Hazen, A., de Bont, A. A., Leendertse, A. J., Zwart, D., de Wit, N. J., de Gier, J. J., \& Bouvy, M. L. (2019). How Clinical Integration of Pharmacists in General Practice has Impact on Medication Therapy Management: A Theory-oriented Evaluation. International journal of integrated care, 19(1), 1. https://doi.org/10.5334/ijic.3291

9. Hawksworth, G. M., Corlett, A. J., Wright, D. J., \& Chrystyn, H. (1999). Clinical pharmacy interventions by community pharmacists during the dispensing process. British journal of clinical pharmacology, 47(6), 695-700. https://doi.org/10.1046/j.1365-2125.1999.00964.x

10. Hill, D., Marr, E., \& Smith, C. (2019). Development of Pharmacist Independent Prescribing Clinics to Treat Opioid Analgesic Dependence in NHS Lanarkshire. Pharmacy (Basel, Switzerland), 7(3), 119. https://doi.org/10.3390/pharmacy7030119
11. Howard, M., Trim, K., Woodward, C., Dolovich, L., Sellors, C., Kaczorowski, J., \& Sellors, J.(2003). Collaboration between community pharmacists and family physicians: lessons learned from the Seniors Medication Assessment Research Trial. Journal of the American Pharmacists Association: JAPhA, 43(5), 566572. https://doi.org/10.1331/154434503322452193

12. Kalman, M. K., Witkowski, D. E., \& Ogawa, G. S. (1992). Increasing pharmacy productivity by expanding the role of pharmacy technicians. American journal of hospital pharmacy, 49(1), 84-89.

https://pubmed.ncbi.nlm.nih.gov/1570872/

13. Keely, E., Tsang, C., Liddy, C., Farrell, B., Power, B., \& Way, C. (2016). Rationale and model for integrating the pharmacist into the outpatient referral-consultation process. Canadian family physician Medecin de famille canadien, 62(2), 111-114. https://www.ncbi.nlm.nih.gov/pmc/articles/PMC4755622/

14. Löffler, C., Koudmani, C., Böhmer, F., Paschka, S. D., Höck, J., Drewelow, E., Stremme, M.,Stahlhacke, B., \& Altiner, A. (2017). Perceptions of interprofessional collaboration of general practitioners and community pharmacists - a qualitative study. BMC health services research,17(1), 224. https://doi.org/10.1186/s12913-017-2157-8.

15. 15. McDonough, R. (2012, September).Challenges to implementation of MTM services. Pharmacy Today, 38. https://www.pharmacytoday.org/article/S10420991(15)31689- 3/pdf

16. McDonough, R. (2016, March).Community pharmacists: retailers or clinicians? Pharmacy Today, 49. https://www.pharmacytoday.org/action/showPdf?pii=S104 2-0991\%2816\%2930159-1

17. Mossialos, E., Courtin, E., Naci, H., Benrimoj, S., Bouvy, M., Farris, K., Noyce, P., \& Sketris, I. (2015). From "retailers" to health care providers: Transforming the role of community pharmacists in chronic disease management. Health policy (Amsterdam, Netherlands), 119(5), 628-639. https://doi.org/10.1016/i.healthpol.2015.02.007

18. Zuckerman, A. D., Carver, A., Cooper, K., Markley, B., Mitchell, A., Reynolds, V. W., Saknini, M., Wyatt, H., \& Kelley, T. (2019). An Integrated Health-System Specialty Pharmacy Model for Coordinating Transitions of Care: Specialty Medication Challenges and Specialty Pharmacist Opportunities. Pharmacy (Basel, Switzerland), 7(4), 163. https://doi.org/10.3390/pharmacy7040163. 